\title{
A PARTICIPAÇÃO DO PACIENTE NA EDUCAÇÃO MÉDICA: REFLEXÕES SOBRE DISCURSOS E EXPERIÊNCIAS NO REINO UNIDO'
}

\author{
PATIENT PARTICIPATION IN MEDICAL EDUCATION: REFLECTIONS ON \\ DISCOURSES AND EXPERIENCES IN THE UNITED KINGDOM
}

Sara Donetto

PhD. Pesquisadora Adjunta,

Faculdade de Enfermagem e Obstetrícia de Florence Nightingale, King's College London.

E-mail: sara.donetto@kcl.ac.uk

\section{Resumo}

Este artigo faz uma revisão geral e levanta questões sobre os discursos dominantes da participação do paciente na educação médica no Reino Unido. Chamando atenção para as dimensões negligenciadas de práticas participativas na pesquisa e pedagogia da educação médica, a discussão dos autores aponta as seguintes questões: quem é o "paciente" nestas práticas de participação? A participação aborda questões relacionadas à epistemologia médica? Os autores sugerem que estas questões deveriam ser centrais para qualquer desenvolvimento na pesquisa e prática em educação médica para abordagens da atenção centradas no paciente e nas relações com pacientes para assim serem traduzidas através de uma retórica política, para a prática clínica do cotidiano. 


\section{Abstract}

This paper provides an overview of, and raises questions about, the dominant discourses of patient participation in medical education in the UK. Drawing attention to neglected dimensions of participatory practices in medical education research and pedagogies, the author's discussion addresses the following questions: Who is the 'patient' in these practices of participation? What does patient participation in teaching activities do to the power relations of medicine? Does participation call into question medical epistemologies? The author suggests that these questions should be central to any developments in medical education research and practice if patient-centred and relationship-centred approaches to care are to translate from policy rhetoric into everyday clinical practice.

Palavras-chave: Educação médica; Participação do paciente no ensino médico; Relação médico-paciente; Reino Unido.

Keywords: Medical Education; Patient involvement in education; Physician-patient relationship; United Kingdom.

Neste artigo, pretendo fornecer uma breve, mas ilustrativa, visão geral dos discursos dominantes da participação do paciente na educação médica no Reino Unido e levantar questões sobre esses discursos (e as práticas às quais se referem e que suscitam), as quais defendo que deveriam estar no centro de todas as abordagens participativas do ensino profissional de saúde. Chamando a atenção para dimensões de práticas participativas que a pesquisa em educação médica atual e pedagogias em grande parte negligenciam, eu discuto as seguintes questões pouco exploradas: quem é 'o paciente' nessas práticas de participação? O que a participação de pacientes em atividades de ensino faz para as relações de poder da medicina? A participação coloca em questão as epistemologias médicas? Eu sugiro que estas questões sejam o centro de desenvolvimentos pedagógicos e de pesquisa em educação médica, se o nosso compromisso para cuidados de saúde mais centrados no relacionamento e no paciente for o de levar da retórica política para a prática clínica diária. Apoio minhas reflexões na minha experiência como estudante de medicina, profissional de medicina e mais extensivamente como pesquisadora na área da prática e educação de cuidados de saúde. Eu me baseio, principalmente, na educação universitária de médicos e no contexto do Reino Unido, mas as questões que levanto aqui se aplicam a toda a gama de profissões da saúde e vão além de contextos nacionais específicos.

\section{O discurso do envolvimento do paciente em política, pesquisa e educação}

Antes de examinar alguns dos aspectos e problemas que caracterizam o envolvimento do paciente em educação médica no Reino Unido, eu gostaria de esclarecer o uso dos termos 'envolvimento' e 'participação' aqui. Na literatura sobre pacientes que assumem um papel 'ativo' (ao contrário de um 'passivo' ou deferente 'fazer o que se manda') em suas consultas com profissionais, o termo 'participação' é visto como colocar o profissional e o não profissional em níveis mais iguais. Cahill apud Thompson ${ }^{1}$ distingue claramente participação "dos conceitos precursores de envolvimento (tarefas básicas, 
frequentemente delegadas) e colaboração (cooperação intelectual) e o conceito final de parceria (joint venture)."1:1299 Embora ciente destas distinções, neste capítulo eu uso os termos envolvimento e participação indistintamente; Eu não penso em envolvimento necessariamente como enfatizando a assimetria de 'recrutamento', mas como um processo de "inserir-se" (do latim in-volvere), tornando-se incluído e engajado em uma atividade em conjunto com outras pessoas, sem referência específica de como ou por quem este engajamento seja iniciado e incentivado.

No Reino Unido, o discurso do envolvimento do paciente na educação médica está incorporado nos mais amplos discursos de pacientes que se envolvem em todas as decisões pertinentes aos seus cuidados clínicos, bem como na concepção e execução de serviços de cuidados de saúde adequados. Esses mais amplos discursos são difundidos e apoiados pela linguagem da política de governo e outras diretrizes e documentos de orientação. Os primeiros gestos em relação à participação mais ativa do paciente nos cuidados da saúde, na política de governo do Reino Unido, podem ser rastreados no final da década de 1980 início da década de 1990." Nas duas décadas seguintes, esses primeiros gestos foram consolidados por uma sequência de documentos políticos publicados sob diferentes governos (conservador 19791997; trabalhista 1997-2010; coligação conservadora-liberal 2010 até hoje ${ }^{2}$ ) que destacaram, entre outras coisas, a necessidade do envolvimento do paciente em decisões sobre seus cuidados, ${ }^{3}$ os benefícios esperados do envolvimento, ${ }^{4}$ e a promessa do governo de 'nenhuma decisão sobre $\mathrm{mim}$, sem $\mathrm{mim}^{\prime} .^{5}$ Com diferentes ênfases e formas de defesa organizada, por exemplo, os Serviços de Assessoria e Defesa do Paciente (PALS), no início da década de 2000; ou as Redes Locais de Envolvimento

\footnotetext{
ii Até o momento que o artigo foi escrito em 2012.
}

(LINKs) no final da década de 2000, o impulso para a participação do paciente na organização dos serviços, nos processos de tomada de decisão com profissionais de saúde e em pesquisa de saúde, foi anunciada igualmente por laboristas, conservadores, liberais-democratas (para um resumo da política-chave e legislação, por exemplo ${ }^{1,6}$ ) Em termos de envolvimento do paciente em decisões e tomada de decisões, orientação oficial foi recentemente publicada pelo Centro Nacional de Prescrição ${ }^{7}$ e pelo Instituto Nacional de Saúde e Excelência Clinica (NICE). ${ }^{8}$ Além disso, o papel dos pacientes como conhecedores 'especialistas' dos mecanismos e opções de tratamento para suas condições médicas foi identificado como um mecanismo fundamental no planejamento e na gestão da crescente carga de doenças crônicas que o Serviço Nacional de Saúde no Reino Unido (NHS) - confrontou. A 'experiência' do paciente tornou-se ligada aos discursos e estratégias de autogestão de doenças crônicas (por exemplo, diabetes, hipertensão) e, nos últimos 10 anos, uma série de 'programas para paciente especialista' foram introduzidos para promover a autogestão e o apoio de pares. ${ }^{9,10, i i i}$

Mais recentemente, o papel do envolvimento ativo de pacientes na concepção e desenvolvimento de pesquisa de cuidado de saúde e na educação de profissionais da saúde tem atraído a atenção de pesquisadores, assim como de organizações profissionais e reguladoras. Em particular, a participação do paciente na educação profissional de saúde parece ser a mais recente estratégia na procura por mais cuidado clínico centrado no paciente.

A educação médica no Reino Unido segue a orientação estabelecida no documento do Tomorrow's Doctors. ${ }^{11,12,13}$ Escolas médicas individuais têm liberdade de escolha na organização de conteúdo

\footnotetext{
iii Consulte também http://www.expertpatients. co.uk/.
} 
e estrutura curricular com o objetivo de atender as recomendações estabelecidas na orientação. Em geral, os currículos de graduação são estruturados para cobrir cinco anos e compreendem uma fase inicial na qual o ensino da ciência básica é dominante, e uma fase posterior na qual a maior parte do tempo curricular é dedicada à aprendizagem clínica. Além disso, a duração do curso se estende em seis anos quando uma então chamada 'Licenciatura Intercalada', uma graduação de um ano de Bacharel em Ciências em algum assunto relacionado é concluída. A distinção entre os anos pré-clínicos e clínicos não é clara, escolas diferentes buscam diferentes graus de integração entre as disciplinas clínicas e não clínicas em todo o currículo. O contato precoce dos alunos com os pacientes e com os ambientes de trabalho é, muitas vezes, incentivado por meio de atividades curriculares dedicadas e estratégias pedagógicas diferentes estão em primeiro plano em diferentes instituições (por exemplo, algumas escolas possuem um componente maior de aprendizagem baseada em problemas, outras privilegiam palestras tradicionais, enquanto outras visam a integração de estratégias pedagógicas diferentes). A maioria das escolas de medicina oferece um conjunto de temas centrais e uma matriz de módulos opcionais a partir da qual os alunos podem escolher de acordo com seus interesses e aspirações.

Como comentei anteriormente, nos últimos dez anos, uma crescente atenção tem sido dedicada pela pesquisa e política em educação médica para o papel dos pacientes na concepção e entrega de currículos de graduação em medicina e na avaliação de estudantes de medicina. Esse interesse está diretamente ligado ao forte compromisso dos cuidados de saúde do Reino Unido para formas de práticas clínica mais centradas no paciente, com base em uma consideração mais séria por parte dos profissionais, das preocupações, preferências e expectativas dos pacientes, e em formas mais colaborativas de tomada de decisão. ${ }^{14,15,16} \mathrm{~A}$ necessidade de envolver os pacientes na educação médica foi, pela primeira vez, mencionada brevemente em 2004 no relatório do Diretor Médico sobre práticas atuais nas escolas médicas do Reino Unido, publicado pelo Department of Health $^{17}$ e, posteriormente destacada, em 2008, no relatório da Associação Médica Britânica, o corpo profissional defendendo e protegendo os médicos do Reino Unido, no texto titulado 'Sobre o papel do paciente em educação médica'. ${ }^{18}$ Baseando-se em estudos de caso de envolvimento de paciente em atividades de ensino, esse relatório apela para a plena participação de pacientes "no desenvolvimento, revisão e implementação de currículos de graduação e pós-graduação em medicina" e no ensino e na avaliação de estudantes de graduação e pós-graduação de medicina. ${ }^{18: 20}$ Ainda mais indicativo do interesse rapidamente propagado de órgãos reguladores oficiais, em 2011, o Conselho Médico Geral emitiu orientações específicas sobre este assunto. O suplemento, um de um conjunto de quatro brochuras $^{\text {iv }}$ destinado a complementar a última edição da Diretriz Nacional para a Educação de Graduação em Medicina no Reino Unido, ${ }^{13}$ explica detalhadamente os aspectos do envolvimento do paciente no ensino, nos quais espera-se que as escolas médicas prestem atenção e respeitem. ${ }^{19}$

Esses discursos reguladores correspondem a um fermento de atividade no conhecimento para educação médica. No início do ano de 2012, interesse acadêmico se uniu em uma conferência de um dia organizada pela Associação para o Estudo da Educação Médica Association for the Study of Medical Education, ASME- na qual educadores,

iv Os outros três tópicos para os quais o GMC emitiu recomendação suplementar são 'avaliação', 'estágios clínicos', e 'desenvolvendo professores e formadores'. 
pesquisadores, e (muito poucosv) pacientes compartilharam experiências sobre os programas existentes, perguntas abertas e as trajetórias para o futuro. Uma visão geral do trabalho publicado sobre a prática atual de participação do paciente no ensino médico é apresentada no relatório de 2011, publicado pela Fundação de Saúde (Health Foundation), ${ }^{20}$ que se baseia no trabalho realizado pela internacional e interdisciplinar Cooperativa de Pesquisa de Pacientes como Educadores, com sede em Vancouver, Canadá. ${ }^{21} \mathrm{O}$ relatório oferece uma discussão das ideias fundamentais e terminologias utilizadas para conceituar e analisar o envolvimento do paciente no ensino. Baseando-se na 'escada' de Tew, Gell e Foster ${ }^{22}$ de envolvimento progressivo do paciente no desenvolvimento e concepção

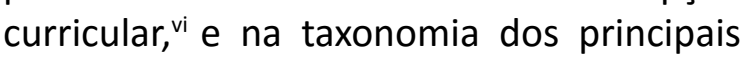
papéis educacionais de Towle et al. ${ }^{21}$ que os pacientes podem ter no currículo de graduação, vii os autores do relatório usam uma taxonomia corrigida para rever estudos de casos de práticas atuais de envolvimento. Esta taxonomia corrigida é organizada em torno de seis 'graus' de envolvimento, que são: 1) pacientes envolvidos na criação de materiais didáticos utilizados pelo corpo docente (por exemplo, caso ou cenário em papel ou eletrônico; materiais do curso;

\footnotetext{
v Lamentavelmente, as taxas de inscrição da conferência são, muitas vezes, inacessíveis para os participantes não acadêmicos ou não clínicos.

vi "De nenhum envolvimento a uma parceria total, em que os pacientes e membros do corpo docente trabalham juntos para tomar decisões sobre o conteúdo e, em conjunto, entregar sessões educacionais." 21:65

vii Taxonomia de Towle et $\mathrm{al}^{21}: 1$ ) cenário em papel ou eletrônico; 2) pacientes padronizados ou simulados; 3) paciente compartilhando sua experiência com os alunos dentro do currículo dirigido pelo corpo docente; 4) professor-paciente(s) envolvidos no ensino e/ou avaliação de alunos; 5) professor-paciente(s) como parceiros iguais na formação do aluno, avaliação e desenvolvimento curricular; e 6) paciente(s) envolvido a nível institucional, além de envolvimento sustentado como professor-paciente(s) em educação, avaliação e desenvolvimento curricular para os alunos. Towle et al. 21:66
}

vídeos); 2) pacientes padronizados ou voluntários em uma clínica; 3) paciente compartilha sua experiência com os alunos dentro de um currículo dirigido pelo corpo docente; 4) professor-paciente(s) estão envolvidos no ensino ou avaliação de alunos; 5) professor-paciente(s) como parceiros iguais na formação do aluno, avaliação e desenvolvimento curricular e/ou pacientes educadores envolvidos em várias áreas do programa; e 6) pacientes envolvidos a nível institucional, além de envolvimento sustentado, como professor-paciente(s) em educação, avaliação e desenvolvimento curricular e/ou pacientes que receberam uma posição formal na instituição. ${ }^{23: 20}$

O relatório reafirma o que já foi antecipado por revisões anteriores, ${ }^{21,24}$ que a literatura sobre o envolvimento do paciente em educação médica é amplamente descritiva e ateórica, em sua maior parte, ou simplesmente descreve as inovações ou apresenta avaliações de curto prazo. Os autores apontam para a revisão radical de Bleakley e Bligh ${ }^{25}$ do papel e da função dos professores na educação médica, mas não começam a explorar com mais profundidade as estruturas teóricas relevantes desses autores ou outros.

Na política e na literatura acadêmica ilustrada até agora, as discussões sobre a participação do paciente no currículo de graduação e em atividades de ensino em particular, são, em grande parte, moldadas dentro de um enfoque avaliativo. Elas examinam, por exemplo, os benefícios desta participação para estudantes (em termos de aumento da confiança e novas ideias), os agentes educativos (em termos de qualidade de ensino e vantagem financeira), e os pacientes (uma sensação de poder e uma melhor compreensão de médicos), e chamam a atenção para as questões éticas importantes levantadas pelos problemas com o consentimento informado dos pacientes e seu direito à confidencialidade. ${ }^{26,23,27}$ É claro que o 
envolvimento do paciente no ensino de graduação deve ser - através de todas as suas fases, desde o planejamento até a implementação - justo, eticamente sólido e sensível ao resultado. No entanto, existe o perigo de que, se incontestado, o caminho para avaliar as práticas atuais, com vista à satisfação de um imperativo baseado em evidências - tal como interpretado por indicadores padronizados de sucesso e resultados mensuráveis - pode ter precedência sobre uma análise mais ampla de como este envolvimento é interpretado e dos sistemas em que está incorporado.

Por isso, agora volto a discutir alguns dos aspectos da participação do paciente no ensino médico que são amplamente negligenciados pelos discursos dominantes de envolvimento e de participação e que têm o potencial de esclarecer aspectos importantes do ensino e aprendizagem em medicina. Sugiro que essas questões pouco exploradas mereçam mais atenção dos pesquisadores e educadores igualmente, se formas mais significativas de colaboração entre profissionais e não profissionais forem alcançadas na educação médica e na prática de cuidados de saúde de forma mais ampla.

\section{'Real', 'padronizado' ou 'simulado': quem é o paciente envolvido no ensino médico?}

Como colocado por Towle et al. ${ }^{21: 65}$ "a linguagem do envolvimento do paciente é confusa e emotiva". No Reino Unido, é comum referir-se a pacientes como 'usuários de serviço' ou, menos frequentemente, 'consumidores', 'fregueses' ou 'clientes'. Questões de identidade e representatividade com relação ao uso do termo 'paciente', no contexto específico de envolvimento do paciente na educação, são brevemente verificadas no relatório acima mencionado. ${ }^{20}$ Embora eu me atenha, neste artigo, ao termo 'paciente' já que é o mais comumente usado nesta área de prática educacional, nesta seção procuro problematizar um aspecto diferente da linguagem que usamos para descrever papéis de ensino diferentes daqueles do aluno e professor/tutor/educador. Em discussões acadêmicas sobre o envolvimento de pacientes na educação médica, é frequente deparar-se com referências a pacientes 'reais', 'atuais', 'simulados' e 'padronizados' sob títulos semelhantes. Ao convidar educadores médicos para compartilhar suas experiências de envolvimento de pacientes em suas atividades de ensino, recebi um pequeno, mas significativo número de descrições que se referiam a pacientes 'reais' e 'simulados' como papéis que eram, de alguma forma, comparáveis e vistos como que pertencendo à mesma área de prática.

É possível, em alguns casos, que o propósito de usar pacientes 'simulados' (atores que representam um paciente), 'padronizados' (indivíduos com ou sem condição médica que treinam para retratar a imagem clínica daquela condição de forma consistente - por exemplo, para provas práticas de alunos), e/ou 'reais' (indivíduos com uma condição médica seja 'atual' ou passada) para a aprendizagem dos alunos possa ser muito semelhante, ou até o mesmo. viii,28,29 Por exemplo, pacientes 'reais' ou 'simulados' podem estar envolvidos na avaliação de desempenho dos alunos durante provas práticas (Exame Clínico Estruturado Objetivo - Objective Structured Clinical Examination/OSCE) ou auxiliar os alunos a praticarem suas entrevistas clínicas. Entretanto, reconhecer que tanto os pacientes 'reais' como

\footnotetext{
viii 0 texto na íntegra deste guia contém 18 páginas e 80 referências e está disponível na: Association for Medical Education na Europa (AMEE), Tay Park House, 484 Perth Road, Dundee DD2 1LR, Reino Unido. E-mail: amee@dundee.ac.uk www.amee.org amee@dundee.ac.uk www.amee.org
} 
atores representando pacientes podem efetivamente contribuir para objetivos específicos de ensino, não justifica desconsiderar os tipos fundamentalmente diferentes de experiências que os pacientes e atores trazem para o ambiente de aprendizagem.

Para ofuscar mais as distinções, pacientes 'reais' podem também ser envolvidos a um grau variável em colaborações com atores. Este envolvimento pode variar desde nenhum envolvimento (por exemplo, quando os atores são munidos de cenários e aconselhados somente por professores do curso) até o envolvimento notável (por exemplo, quando pacientes e atores trabalham juntos nos cenários e no feedback das dramatizações, como no caso da Imperial College Medical School, em Londres. ${ }^{19}$ Esses extremos não estão em uma escala de valor, no sentido de que a abundância de envolvimento não é automaticamente mais desejável do que nenhum envolvimento. Em vez disso, graus diferentes de colaboração entre atores, pacientes e professores clínicos são adequados para diferentes tipos de ensino e aprendizagem (por exemplo, cenários muito simples, com o objetivo de iniciar os alunos em dramatização e comunicação clínica podem ser adequadamente produzidos pelos professores clínicos sem qualquer outra contribuição, enquanto cenários com o objetivo de desafiar as suposições dos alunos sobre uma condição clínica ou uma forma de interação podem se beneficiar de uma ampla contribuição do paciente). Aqui, gostaria de chamar a atenção para o fato de que, ao usar essas definições como abreviação para referir-se aos papéis sociais e pedagógicos específicos, corremos o risco de obscurecer o fato de que as experiências das pessoas, o seu posicionamento social e as relações de poder em que elas se envolvem são suscetíveis de ser muito diferentes para esses diferentes colaboradores. Referir-se, genericamente, comoé ocaso do aditamento de orientação Tomorrow's Doctors a 'pacientes, indivíduos leigos e ao público' como agrupamento difuso de 'pacientes ou pacientes atuais; pacientes experientes; cuidadores, advogados e funcionários de apoio; pacientes padronizados ou simulados; pacientes virtuais; pessoas leigas ou 'o público'; grupos ou representantes de pacientes; organizações do setor voluntário ou comunitário local ${ }^{19: 4}$ pode ser um atalho pragmático para um documento político, mas precisamos garantir que as análises cuidadosas dos papéis e significados estejam disponíveis em outros lugares. Da mesma forma, não há nada de errado em praticar o pensamento clínico de alguém com o auxílio de cenários de caso que podem envolver vinhetas escritas, manequins de simulação, atores ou pacientes 'reais', enquanto todos nós educadores, estudantes, pacientes, atores e praticantes prestamos atenção aos efeitos intencionais e não intencionais da implantação de diferentes recursos e atuação de diferentes colaborações. Nos discursos dominantes atuais de participação de paciente no ensino, há um perigo de falhar ao fazer uma distinção sobre quem está participando e de que maneira.

Nós precisamos garantir a revisão constante da linguagem que usamos para descrever e compartilhar nossas experiências de/com práticas participativas. O próprio termo 'paciente simulado' acredito pode ser visto como indicativo de uma abordagem nãocrítica para colaborações entre pacientes e profissionais. Eu mais pedantemente argumentaria que a expressão 'paciente simulado' pode sugerir uma facilidade de comparação entre a experiência real e simulação/atuação que é improdutiva e possivelmente enganosa. Isso pode, inadvertidamente, ofuscar as fronteiras entre as histórias da 'vida real' e as representações escritas de histórias da vida real, ocultando assim as qualidades específicas da experiência da doença de cada pessoa e da atenção que os profissionais devem estar dando a essas qualidades. 
Pacientes como coeducadores:

transformando as relações sociais da medicina?

Um aspecto fundamental da participação do paciente no ensino médico que, com poucas exceções significativas, por exemplo, Bleakley, Bligh e Browne ${ }^{30}$ é amplamente ignorado no conhecimento da educação médica é a análise das formas em que estas iniciativas podem desafiar, transformar ou reforçar as relações de poder predominantes em encontros de cuidados de saúde e construções associadas de pacientalidade, experiência relacionada com a saúde e os papéis de saúde. ${ }^{i x}$ Por exemplo, raramente pesquisadores (ou educadores, para esse assunto) exploram as interações reais que ocorrem durante as sessões que se beneficiam da contribuição do paciente, a medida em que os pacientes podem tornar-se profissionalizado no seu papel de 'especialistas' ou como 'professores', ou o local que essas sessões ocupam no contexto do currículo. A escassez de uma visão crítica das práticas de participação levanta questões sobre a medida em que a promoção de parcerias e colaboração nos cuidados de saúde, de forma mais geral, se baseia em entendimentos restritos de participação e hegemonia médica tradicional. Se queremos que os médicos sejam reflexivos e críticos sobre sua prática e suas interações com pacientes, devemos garantir que nós sejamos reflexivos e críticos em nosso ensino, que examinemos de perto os lugares que os pacientes, professores e alunos ocupam nos processos de aprendizagem nos quais estão envolvidos.

\footnotetext{
ix Em 2009, uma revisão feita por Jha et al..$^{24}$ destacou que a literatura acadêmica sobre o envolvimento do paciente na educação continha pouca exploração do possível impacto da profissionalização dos pacientes como educadores nas relações de poder dentro da medicina. Entretanto, esses tipos de estudos continuam raros.
}

Uma das formas mais comuns de envolvimento do paciente observa pacientes fornecendo aos alunos um relato de sua experiência da doença e de suas experiências dentro do serviço de saúde. Sem dúvida, as considerações dos pacientes trazem para o ensino uma perspectiva que nenhuma outra forma de ensino pode oferecer; elas contribuem um ponto de vista sobre os cuidados médicos com os quais os alunos devem se envolver, se quiserem ganhar profundidade em sua compreensão de como a prática clínica funciona. Apesar de importante, no entanto, esta contribuição é também limitada. Nessa abordagem para contribuição dos pacientes, eles continuam sendo 'portadores', não mais dos sinais e sintomas físicos, mas de histórias e cenários da 'vida real'. Os pacientes são convidados a ensinar, informados sobre os aspectos práticos disso, possivelmente treinados para sua função e, finalmente, introduzidos nas audiências de alunos de medicina. Eles são questionados sobre sua experiência e suas visões são ouvidas. Este é um passo enorme na direção de uma prática médica mais respeitosa, mas é um passo que se mantém confinado dentro de uma construção de pacientes como 'recursos instrucionais'. ${ }^{31}$

Nesta e em outras formas de participação do paciente no ensino

a comunicação entre o aluno e o paciente [...] tende a ser de uma via, com o aluno iniciando as questões e o paciente colocado no papel de passivo de um objeto de investigação para alunos. 0 estudante de medicina aprende com o paciente, mas o que ele aprende é amplamente filtrado e controlado pelo professor clínico e outros membros da equipe de cuidados de saúde. ${ }^{30: 195}$

Uma coisa é incluir pacientes como 'pessoas com autoridade' em atividades de ensino, oferecendo uma importante 
e, por outro lado, deficiente perspectiva sobre a doença e cuidado, e outra coisa completamente diferente é pensar nos pacientes como potenciais colaboradores na formação de médicos. A abordagem que eu, como Bleakley e Bligh, ${ }^{25}$ gostaria de ver aprovada é aquela que desafia as relações sociais da medicina, fomentando pelo menos em alguns espaços e formatos de aprendizagem relações educativas nas quais os pacientes pudessem ser vistos como educadores, os alunos como aprendizes e professores de medicina como facilitadores de aprendizagem. ${ }^{25,32}$ Esta configuração diferente dos papéis tem o potencial de desafiar seriamente a ortodoxia de poder da educação médica. Para Bleakley, Bligh e Browne ${ }^{30}$, por exemplo, isso pode atrapalhar a maneira habitual de se relacionar com os pacientes, como se fossem quebra-cabeças de diagnóstico a serem resolvidos:

Como o aluno se orienta aos pacientes, apoiado pelo professor clínico agora atuando como facilitador, pode ser que o aluno se torne mais sensível à narrativa do paciente e menos inclinado a traduzir instantaneamente a narrativa em um problema técnico a ser resolvido. ${ }^{30: 195}$

Em vista disso, também devemos questionar discursos e práticas para entender melhor de que forma as novas relações de ensino e aprendizagem podem afetar a modelagem de papel na aprendizagem clínica. $E$, por modelagem de papel, refiro-me aos exemplos que os professores constituem no seu papel de médicos, mas também àqueles que prestam no seu papel de educadores (para uma discussão mais ampla sobre o papel das relações professor-aluno na educação médica, consulte, por exemplo $33,34)$. Poderíamos estar perguntando, por exemplo: que mensagem os professores transmitem sobre os pacientes e a participação dos pacientes quando eles são os principais diretores dessa participação? Ou se eles deixam de examinar o que eles como médicos e como professores também estão aprendendo com esse processo ${ }^{x}$

\section{Desafiando epistemologias médicas?}

A participação do paciente no ensino médico gera possibilidades para análise e contestação ao que conta como conhecimento na medicina? Quando explorei a percepção crítica (uma adaptação da consciência crítica do Paulo Freire ${ }^{35,36, x i}$ ) dos alunos de medicina para minha pesquisa de doutorado, examinei como os alunos atuam distinções claras entre as 'sólidas' formas de conhecimento - ou seja, aquelas da ciência médica, de causas e efeitos, de tratamentos farmacológicos - e aquelas 'amenas', ou seja, relacionadas, por exemplo, à comunicação (descritas como 'suaves', 'leves' ou 'brandas'), pensamento ético e cuidado da saúde mental. Após argumentar, continuo afirmando que esta categorização do conhecimento é sintomática de uma predominância impregnada e não reconhecida das epistemologias positivistas na educação médica ${ }^{37,38}$ que o currículo não pareceu desafiar de forma significativa. Essas categorizações são improdutivas, por diversas razões, mas de particular relevância aqui é o fato de que elas reproduzem formas de pensamento e compreensão dos cuidados de saúde que não conseguem se envolver com a interdependência entre as diversas dimensões das experiências da doença, para as quais os médicos são chamados a prestar atenção.

\footnotetext{
× Não há, praticamente, nenhuma discussão na literatura acadêmica sobre como o envolvimento do paciente no ensino contribui para o desenvolvimento pessoal e profissional dos educadores.

xi Embora use uma terminologia diferente para evitar sugerir uma sobreposição completa com a teoria de Freire em sua totalidade.
} 
$\mathrm{Na}$ análise de abordagens de pesquisa dominantes para práticas de envolvimento do paciente nas decisões de tratamento que realizei com Alan Cribb, sugerimos que "nem as práticas, nem o estudo de envolvimento do paciente nos parecem em última análise, ser viável metodologicamente ou eticamente a não ser sustentados por algum compromisso significativo com o 'envolvimento epistêmico'". ${ }^{39: 910}$ Por 'envolvimento epistêmico' nos referimos a "levar a sério as perspectivas dos pacientes, não como uma fonte extra de dados para alimentar as epistemologias clínicas, mas como uma fonte de enquadramentos e visões epistemologicamente alternativas." O envolvimento epistêmico exige, em outras palavras, uma habilidade por parte de todos os atores sociais envolvidos, mas em particular aqueles nos papéis profissionais, que possuem uma responsabilidade de orientar o curso de ação, para envolver-se genuinamente com maneiras desconhecidas e potencialmente desconfortáveis de enquadrar a experiência. No intuito de mover-se, na prática clínica tanto quanto na educação, além de simplesmente "utilizar a retórica do 'paciente especialista' enquanto ainda construindo o paciente no que é fundamentalmente um papel passivo, ignorante e inativo" ${ }^{6}$, a participação do paciente requer médicos, educadores e alunos que sejam capazes de pensar de forma flexível sobre o conhecimento e o saber.

No contexto aqui discutido, envolvimento epistêmico significa garantir que a participação do paciente no ensino seja baseada em entendimentos negociados de quais são seus objetivos e limitações. O consenso sobre quais são os papéis e responsabilidades dos pacientes, em cada configuração específica, deve ser alcançado através da negociação de diferentes agendas, as quais devem incluir as agendas dos pacientes, educadores e alunos. Uma abordagem crítica aos papéis dos pacientes como educadores significa garantir que os educadores, pacientes e pesquisadores prestem atenção à mensagem intencional e não intencional que a prática de ensino carrega. Isso significa também fazer o tempo e o espaço para questionamento epistemológico, para os alunos começarem a se envolver com a forma que o conhecimento é produzido e com o que conta como verdade. É essencial, nesse processo, evitar que o ensino baseado em narrativas de experiência de vida e doença seja percebido pelos alunos como mero acessório ao ensino da ciência médica. Ou seja, incentivar uma linguagem de integração e interdependência, em oposição a uma linguagem de complementaridade baseada em distinções precisas.

\section{Pesquisando a participação do paciente no ensino: influenciando a prática além da normatividade baseada em evidência}

Existem, pelo menos, duas principais razões por que na prática educacional, mas ainda mais na pesquisa médica educacional nós devemos prestar mais atenção às questões que levantei nas seções anteriores. A primeira é uma razão muito prática que se refere ao entendimento profundo do que pretendemos ensinar, o que realmente ensinamos e como o ensinamos; a segunda tem a ver com ser capaz de traçar direções coerentes para estratégias de pesquisa, política e educação.

A avaliação de eficácia e sustentabilidade, que é um tema tão proeminente na pesquisa e prática atuais, não nos oferece por si própria uma imagem suficientemente rica do processo de aprendizagemeensino.Senosso realobjetivo for desenvolver profissionais que possam praticar de maneiras centradas no paciente 
ou melhor ainda, centradas nas relações, ${ }^{40,41}$ que possam refletir sobre sua prática, serem sensíveis às circunstâncias específicas e possuírem visões críticas na complexidade dos encontros clínicos, então as leituras restritas do que conta como 'evidência' em práticas baseadas em evidência simplesmente não será suficiente. ${ }^{42,43}$ A necessidade de avaliar a eficácia e os fatores determinantes para a eficácia, a fim de formular decisões de financiamento e sustentabilidade responsáveis, na verdade, exige mais do que exclui a necessidade de investigar e esclarecer os mecanismos mais sutis de processos de aprendizagem. Onde análises deste tipo existam em outras áreas disciplinares, como a sociologia, filosofia, antropologia -para mencionar algumas áreas em que os acadêmicos se interessaram em educação profissional em saúde - deve-se permitir mais espaço para elas nos fóruns oficiais de educação médica. Atualmente, estudos aprofundados, por exemplo, das narrativas, discursos e negociações de poder que a participação no ensino acarreta, legitima ou desafia são muito raros e difíceis de localizar. Estas análises mais sociologicamente esclarecidas são mais propensas de serem realizadas na pesquisa que explora questões mais amplas de engajamento do paciente e do público (abreviado como PPE ou PPI - envolvimento do paciente e do público no Reino Unido), com impulso limitado na literatura de educação médica. ${ }^{44,45}$

A segunda razão para fazer perguntas mais críticas sobre a participação do paciente na educação médica é conseguir alguma coerência entre objetivos declarados educacionais, trajetórias políticas, práticas pedagógicas e estratégias de pesquisa. Há tensões e inconsistências no ensinar abordagens de cuidados com base no diálogo, na empatia e foco no paciente, predominantemente através de pedagogias baseadas nas abordagens comportamentais de 'instigação.' ${ }^{32} \mathrm{Da}$ mesma forma, há tensões e contradições no avaliar e explorar práticas de participação através de abordagens de pesquisa que deixam pouco espaço para considerações de profundidade, desenvolvimento de longo prazo e complexidade do sistema. A fim de se comprometer significativamente com o tipo de perguntas que estou chamando a atenção neste momento, precisamos de culturas acadêmicas e sistemas de financiamento que apoiem completamente os entendimentos mais amplos do que conta como evidência na prática baseada em evidências. Em níveis institucionais, precisamos de apoio para 'programas coordenados e sustentados que trazem a voz e experiência autêntica e autônoma do paciente para a educação dos profissionais de saúde'. ${ }^{46: 501}$ No entanto, quando esses programas já existem ou estão sendo implementadas como tentativa, ${ }^{\text {xi }}$

\footnotetext{
xii Exemplos de reconfiguração inovadora de relações entre paciente e profissional da saúde na educação podem ser encontrados no Reino Unido e no Canadá. A escola de medicina Leeds, no Reino Unido, busca o envolvimento da comunidade em vários componentes do ensino de graduação, com base em 'uma filosofia subjacente de um contrato social entre a Leeds School of Medicine e a comunidade local'. 20:54; enquanto a University of Warwick apóia uma 'Parceria para Ação de Pesquisa e Ensino do Usuário da Universidade' (UNTRAP - University User Teaching and Research Action) que visa garantir que os membros sejam 'valorizados como membros de qualquer grupo de trabalho e não apenas como 'usuário do serviço / perito em experiência de paciente'; recebam apoio adequado, incluindo o acesso à informação e instalações de TI e oportunidades de treinamento; e pagos e reembolsados a uma taxa justa para todas as atividades que realizem ${ }^{20: 56}$. No Canadá, a McMaster University ofereceu às pessoas com HIV a oportunidade de realizar 'um extenso programa educacional que forneceu a elas mais do que contar uma história e os ajudou a facilitar a aprendizagem'. ${ }^{20: 47}$ Finalmente, com uma abordagem bastante radical para transformar as relações dominantes dentro da medicina, a University of British Columbia, em Vancouver, está pilotando um programa de 'mentores de saúde', através do qual as pessoas que sofrem de doenças crônicas encontram pequenos grupos de alunos da profissão de saúde (incluindo estudantes de medicina) em uma base regular (duas vezes por semestre para um total de 6 encontros ao longo de 16 meses) e na ausência de professores para discutir temas sugeridos ou emergentes (após uma reunião
} 
também precisamos de apoio para as formas de análise e avaliação que sejam, por elas próprias, colaborativas e capazes de gerar considerações valiosas de prática ou seja, considerações que incluam várias lentes, camadas de significado e graus de complexidade. Finalmente, precisamos também de compromisso acadêmico para a fertilização cruzada através das fronteiras linguísticas e geográficas. O ensino e a prática educacional médica têm muito a aprender com as experiências práticas e conhecimentos teóricos de contextos não

inicial adicional para conhecer uns aos outros e estabelecer regras básicas) (consulte http://www.chd. ubc.ca/dhcc/healthmentors/) expressados em inglês, e as experiências de governança participativa no Brasil são um exemplo pertinente em matéria de práticas colaborativas. ${ }^{47,48}$ Oportunidades de intercâmbio e fertilização cruzada devem ser promovidas e sustentadas por instituições de ensino superior não apenas através do financiamento de programas de intercâmbio de estudantes e pesquisadores, mas também através de estruturas acadêmicas e oportunidades que apoiem o multilinguismo e a compreensão cultural (inclusive política) como fundamentais para o desenvolvimento da educação profissional em qualquer contexto.

\section{Referências}

1. Thompson AGH. The meaning of patient involvement and participation in health care consultations: a taxonomy . Social science \& medicine, 64(6); 2006: 1297-1310.

2. Department of Health. Working for patients. London: Department of Health; 1989.

${ }^{3}$. Department of Health - NHS Executive. Patient partnership building a collaborative strategy. London: Department of Health; 1996.

${ }^{4}$. Department of Health. Saving lives: our healthier nation. London: Department of Health; 1999.

5. Department of Health. Equity and excellence: liberating the NHS. London: Department of Health; 2010.

6. Greenhalgh T, Humphrey C, Woodard, F. User involvement in health care. Oxford: Wiley-Blackwell; 2011.

7. Clyne W, Granby T, Picton C. A competency framework for shared decision-making with patients: achieving concordance for taking medicines. Keele, UK: National Prescribing Centre Plus; 2007.

${ }^{8}$. National Institute for Health and Clinical Excellence. Medicines adherence: involving patients in decisions about prescribed medicines and supporting adherence - quick reference guide. London: NICE; 2009.

9. Department of Health. Patient and public involvement in the new NHS: report. London: Department of Health; 1999. 


\section{Artigo Original}

${ }^{10}$. Department of Health. The expert patient: a new approach to chronic disease management for the 21st century. London: Department of Health; 2001.

${ }^{11}$. General Medical Council. Tomorrow's doctors: recommendations on undergraduate medical education. London: GMC; 1993.

12. General Medical Council. Tomorrow's doctors: London: GMC; 2003.

13. General Medical Council. Tomorrow's doctors: outcomes and standards for undergraduate medical education. London: GMC; 2009.

14. Mead N, Bower P. Patient-centredness: a conceptual framework and review of the empirical literature. Social science \& medicine, 51(7); 2006: 1087-1110.

15. Charles C, Gafni A, Whelan T. Shared decision-making in the medical encounter: What does it mean? (or it takes at least two to tango). Social science \& medicine, 44(5); 1997: 681-692.

${ }^{16}$. Charles C, Gafni A, Whelan T. Decision-making in the physician-patient encounter: Revisiting the shared treatment decision-making model. Social science \& medicine, 49(5); 1999: 651661.

17. Department of Health. Medical schools: Delivering the doctors of the future. London: Department of Health; 2004.

${ }^{18}$. British Medical Association. Medical Education Subcommittee. Role of the patient in medical education. London: BMA; 2008.

19. General Medical Council. Patient and public involvement in undergraduate medical education: advice supplementary to Tomorrow's Doctors (2009). London: GMC; 2011.

${ }^{20}$. Spencer J et al. Can patients be teachers? involving patients and service users in healthcare professionals' education. London: The Health Foundation; 2011.

${ }^{21}$. Towle A et al. Active patient involvement in the education of health professionals. Medical education, 44(1); 2010: 64-740.

22. Tew J, Gell C, Foster S. Learning from experience: involving service users and carers in Mental Health Education and Training. NIMHE/Trent Workforce Development Corporation; 2004.

23. SPENCER, J. et al. Patient-oriented learning: a review of the role of the patient in the education of medical students. Medical Education, 34:; 2000: 851-857.

${ }^{24}$. Jha $V$ et al. Strategies and interventions for the involvement of real patients in medical education: A systematic review. Medical Education, 43(1); 2009: 10-20.

25. Bleakley A, Bligh J. Students learning from patients: let's get real in medical education. Advances in Health Sciences Education, 13(1); 2008: 89 -107.

${ }^{26}$. Howe A, Anderson J. Involving patients in medical education. British Medical Journal, 327; 2003: 326-328.

27. Wykurz G, Kelly D. Developing the role of patients as teachers: literature review. British Medical Journal, 325(7368); 2002: 818-821. 


\section{Artigo Original}

${ }^{28}$. Collins J P, Harden RM . AMEE medical education guide n. 13: real patients, simulated patients and simulators in clinical examinations. Medical Teacher, 20(6); 1998: 508-521.

29. Barrows HS. An overview of the uses of standardized patients for teaching and evaluating clinical skills. Academic Medicine, 68(6); 1993: 443-451.

30. Bleakley A, Bligh J, Browne J. Medical education for the future: identity, power and location. London: Springer; 2011.

31. Bell $\mathrm{K}$ et al. When only the realthing will do: Junior medical students' learning from real patients. Medical Education, 43(11); 2009:1036-1043.

32. Donetto S. Medical students' views of power in doctor-patient interactions: the value of teacher-learner relationships. Medical Education, 44(2); 2010: 187-196.

${ }^{33}$. Haidet $P$, Stein HF. The role of the Student-Teacher relationship in the formation of physicians. Journal of General Internal Medicine, 21(Supl.1); 2006: 16.

${ }^{34}$. Tiberius, R. G.; Sinai, J.; Flak, E. A. The role of teacher-learner relationships in medical education. In: Norman GR, Van Der Vleuten C, Newble D. (Ed.). International handbook of research in medical education Dordrecht, Netherlands: Kluwer Academic Publisher; 2002: 463-497.

${ }^{35}$. Freire P. Pedagogy of the oppressed. London: Penguin; 1996.

36. Freire P. Education for critical consciousness. London: Continuum; 2007.

37. Donetto S. Medical students and patient-centred clinical practice: the case for more critical work in medical schools. British Journal of the Sociology of Education, 33(3); 2012: 431-449.

${ }^{38}$. Donetto S, Cribb A. Beyond binaries in medical knowledge: a call for epistemological reflexivity. Interface-Comunicação, Saúde, Educ Botucatu, 15(38); 2011: 924-925.

39. Donetto S, Cribb A. Researching involvement in health care practices: interrupting or reproducing medicalization? Journal of Evaluation in Clinical Practice, 17(5); 2011: 907-912.

${ }^{40}$. Beach MC, Inui T. A constructive reframing. Journal of General Internal Medicine, 21(supl.1); 2006: 3-8.

${ }^{41}$. Tresolini CP, Pew-Fetzer Task F. Health professions education and relationship-centered care: report of the Pew-Fetzer task force on advancing psychosocial health education. San Francisco, CA: Pew Health Professions Commission; 1994.

42. Biesta G. Why "what works" won't work: evidence-based practice and the democratic deficit in educational research. Educational Theory, 57(1); 2007: 1-22.

${ }^{43}$. Hammersley M. Some questions about evidence-based practice in education. Paper presented at the Symposium on Evidence-Based Practice in Education at the Annual Conference of the British Educational Research Association, University of Leeds, England, Sept . 2001.

${ }^{44}$. Hogg C, Williamson C. Whose interests do lay people represent? Towards an understanding of the role of lay people as members of committees. Health Expectations, 4(1); 2001: 2-9. 
Artigo Original

${ }^{45}$. Sinding $\mathrm{C}$ et al. Of time and troubles: Patient involvement and the production of health care disparities. Health, 16(4); $2012: 400-417$.

${ }^{46}$. Towle A, Godolphin W. A meeting of experts: the emerging roles of non-professionals in the education of health professionals. Teaching in Higher Education, 16(5); 2011: 495-504.

47. Avritzer L. New public spheres in Brazil: local democracy and deliberative politics. International Journal of Urban and Regional Research, 30(3); 2006:623-637.

48. Gandin LA, Apple MW. Challenging neo-liberalism, building democracy: Creating the citizen school in Porto Alegre, Brazil. Journal of Educ Policy, 17(2); 2002: 259-279. 